TITLE:

\title{
Modal parameter identification of short span bridges under a moving vehicle by means of multivariate $A R$ model
}

\section{$\operatorname{AUTHOR}(\mathrm{S}):$}

Kim, C. W.; Kawatani, M.; Hao, J.

\section{CITATION:}

Kim, C. W....[et al]. Modal parameter identification of short span bridges under a moving vehicle by means of multivariate AR model. Structure and Infrastructure Engineering 2012, 8(5): 459-472

ISSUE DATE:

2012-05

URL:

http://hdl.handle.net/2433/172227

\section{RIGHT:}

(C) 2012 Taylor \& Francis; この論文は出版社版でありません。引用の際 には出版社版をご確認ご利用ください。; This is not the published version. Please cite only the published version. 


\title{
Modal parameter identification of short span bridges under a moving vehicle by means of multivariate AR model
}

\author{
C.W. KIM ${ }^{*}$, M. KAWATANI + , AND J. HAO \\ $\dagger$ Kyoto University, Department Civil and Earth Resources Engineering, Kyoto 615-8540, Japan \\ $\$$ Kobe University, Department of Civil Engineering, Kobe 657-8501, Japan \\ $\S$ General Consulting Department, Construction Project Consultants, Inc, Tokyo, Japan
}

\begin{abstract}
This study is intended to investigate the feasibility of health monitoring of short span bridges based on modal parameter identification using the vibration data of the bridge induced by a moving vehicle. The idea of this study using the traffic-induced vibration of short span bridges which is a kind of non-stationary process is that the modal parameters identified repeatedly under a given moving vehicle provide a pattern which may provide useful information to decide current health condition of bridges. The autoregressive (AR) model is adopted for identifying modal parameters such as frequency and damping characteristics of the bridges. Mode shapes of bridges are obtained using multivariate AR model. Feasibility of the modal parameter identification for health monitoring of short span bridges is observed through a moving vehicle laboratory experiment. Pattern changes of identified parameters are observable by comparing the identification results between intact and damaged girders, which encourages the use of the method for long term health monitoring even for short span bridges.
\end{abstract}

Keywords: Bridge health monitoring; Moving vehicle laboratory experiment; Modal parameter identification; Autoregressive model; Short span bridge; Traffic-induced vibration

\section{Introduction}

Minimizing the safety risk of bridge structures has become an important technical issue. Bridge health monitoring (BHM) at a global level using dynamic system parameters thus has been one of the most important approaches, and also has been intensively studied (e.g. Doebling et al. 1996). The basic idea behind BHM using the dynamic system parameters is that frequency and damping characteristics as well as mode shapes may provide useful information for the current health condition of bridges. The fundamental concept of this technology is that modal parameters are functions of a structure's physical properties. Therefore, a change in physical properties, such as reduced stiffness resulting from damage, will detectably change these modal properties (e.g. Friswell \& Mottershead 1994). Of course, applying sensors around expected or suspected damage substructures is one of the best approaches to detect damage. This is only effective, however, if the bridge structure has well defined damage models. For real bridge structures it is difficult to define a damage model differently from other structures such as automobiles, aerial vehicles, etc. Therefore, most precedent studies focusing on bridge health monitoring have specifically examined the global change of modal properties and quantities of bridge structures.

Since the 1970s, the use of state-space models for modal parameter identification in time-domain has been increasing and also has yielded new approaches. Gersch et al. (1973), for example, used the time series of an Autoregressive Moving Average (ARMA) process to describe the random response of a vibrating structure to a white noise excitation. Shinozuka et al. (1982) obtained a second-order ARMA model to represent a vibrating structure in order to identify the structural parameters directly. Hoshiya and Saito (1984) included the parameters to be identified as additional state variables in the state vector using extended Kalman filter. These approaches regard the ambient vibration responses as random process of

\footnotetext{
* Corresponding author. Email: kim.chulwoo.5u@kyoto-u.ac.jp
} 
ARMA. Estimating the coefficients of ARMA model is a kind of nonlinear approach because both of the coefficients relating to AR and MA processes are unknown variables. Fortunately, the AR model with infinite order is equivalent to the ARMA model, which means that one can express the responses of a linear system subjected to white-noise input using the AR model with sufficient large order (Wang \& Fang 1986).

All of the methods assume white noise excitations. In practice, however, ambient vibration responses observed in operation, which cannot be white noise excitations, are used in modal parameter identification (e.g., Hoshiya \& Saito 1984, He \& Roeck 1997). For long span bridges, wind and traffic-induced vibrations are operational dynamic sources. On the other hand, for short span bridges which are insensitive (or sometimes impassive) to the wind load, the normal traffic excitations are important dynamic sources. However, the traffic-induced vibration is a kind of non-stationary process (Kim et. al 2005, Kim \& Kawatani 2008) that becomes stronger with decreasing span length. Despite of non-stationary characteristic of traffic-induced vibrations of short span bridges, the single or normal traffic excitations are still attractive dynamic sources if we can utilize them for health monitoring of short span bridges.

The idea of this study even using the traffic-induced vibration of short span bridges for their modal identification is that the modal parameters identified repeatedly under a given moving vehicle can provide a pattern or even a statistical one, which may give useful information to decide current bridge condition. For the short span bridges in local areas where we cannot expect vibrations whenever we want because of their light traffic volumes, an inspection car may be a solution to excite the bridge. Cost-effectiveness is also an important factor to be considered in developing a health monitoring system for short span bridges because a large number of those short span bridges are located in local areas and applying health monitoring system even to the bridge on local areas needs a stupendous budget.

This paper covers feasibility investigations for health monitoring of short span bridges by means of modal parameter identification using traffic-induced vibrations through a moving vehicle laboratory experiment. The experiment is conducted for both intact and damage girders. Considering costeffectiveness this study uses limited numbers of sensors. Moreover how vehicle speed and type affect identified results of modal parameters is an important matter to investigate feasibility of the inspection car for health monitoring of short span bridges, because dynamic responses of short span bridges are easily affected by vehicles' dynamic characteristics as previously described. Therefore this study also examined effects of vehicle speed and type to the modal parameter identification.

Modal parameters are identified using the autoregressive (AR) model derived from the state equation of a bridge structure. Usually, for an engineering decision, the more the data the better the results are, which is also true for deciding health condition of bridges. Therefore, both singlevariate autoregressive (SAR) and multivariate autoregressive (MAR) models are adopted in this study, because the SAR model provides more identified parameters than those by the MAR model: the SAR model identifies those modal parameters of every single sensor while the MAR model provides modal parameters of a system (or entire sensor group of a bridge). Noteworthy point is, on the other hand, that the SAR model is not applicable to estimating the mode vector which can be obtained by the MAR model. Therefore, a two-step approach is applied: the first step identifies modal frequencies and damping using a SAR model; and identifying mode shapes by means of MAR model is the second step. MATLAB (1997) is used to code the algorithm.

\section{Time-series model of a dynamic system}

The MAR model of a structural system is derived for a general form, because the same procedure is easily applicable to the derivation of the SAR model.

\subsection{State-space equation}

The equation of motion for a bridge is described as

$$
\mathbf{m}_{b} \ddot{\mathbf{w}}(t)+\mathbf{c}_{b} \dot{\mathbf{w}}(t)+\mathbf{k}_{b} \mathbf{w}(t)=\mathbf{f}(t)
$$


where, $\mathbf{m}_{b}, \mathbf{k}_{b}, \mathbf{c}_{b} \in \mathbf{R}^{n \times n}$ respectively indicate the mass, damping, and stiffness matrices of the bridge. $n$ denotes the degrees-of-freedom of the bridge model. $\mathbf{w}(t) \in \mathbf{R}^{n}$ and $\mathbf{f}(t) \in \mathbf{R}^{n}$ denote the displacement and external force vectors respectively. The state vector $\mathbf{x}(t)$ for the equation of motion of a bridge is definable as

$$
\mathbf{x}(t)=\left[\begin{array}{c}
\mathbf{w}(t) \\
\dot{\mathbf{w}}(t)
\end{array}\right]
$$

If $\mathbf{y}(t) \in \mathbf{R}^{m}$ denotes output of the bridge structure taken from $m$ observation points, then the corresponding state equation of a continuous-time system is described as

$$
\begin{aligned}
& \dot{\mathbf{x}}(t)=\mathbf{A x}(t)+\mathbf{B f}(t) \\
& \mathbf{y}(t)=\mathbf{C x}(t)
\end{aligned}
$$

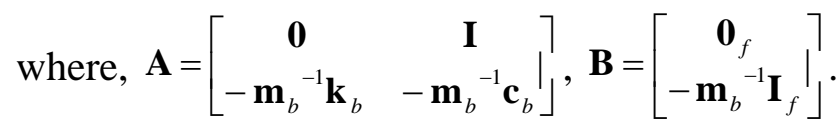

In Eqs.(3) and (4), $\mathbf{A} \in \mathbf{R}^{2 n \times 2 n}, \mathbf{B} \in \mathbf{R}^{2 n \times n}$ and $\mathbf{C} \in \mathbf{R}^{m \times 2 n}$ respectively denote system, input influence and output influence matrices. Especially, $\mathbf{C}$ is a transformation matrix mapping the position of system degrees of freedom with measured outputs which consists of zero or one. $\mathbf{I}_{f} \in \mathbf{R}^{n \times l}$ is the index matrix for the location of $l$ inputs. $\mathbf{0}_{f} \in \mathbf{R}^{n \times(n-l)}$ denotes the null matrix.

For gradual change of $\mathbf{f}(\tau)$ within a equally spaced time interval such as $t_{k} \leq \tau \leq t_{k+1}\left(=t_{k}+\Delta t\right), \mathbf{f}(\tau)$ is constant as

$$
\mathbf{f}(\tau)=\mathbf{f}(k), \text { for } t_{k} \leq \tau \leq t_{k+1}
$$

Then the discrete form solution is writable as

$$
\mathbf{x}(k+1)=\overline{\mathbf{A}} \mathbf{x}(k)+\overline{\mathbf{B}} \mathbf{f}(k)
$$

where $\overline{\mathbf{A}}=e^{\mathbf{A} \Delta t}$ and $\overline{\mathbf{B}}=\int_{t_{k}}^{t_{k+1}} e^{\mathbf{A}\left(t_{k+1}-\tau\right)} \mathbf{B} d \tau=\left(e^{\mathbf{A} \Delta t}-\mathbf{I}\right) \mathbf{A}^{-1} \mathbf{B}$.

The measurement (or observations) is shown in Eq. (7).

$$
\mathbf{y}(k)=\overline{\mathbf{C}} \mathbf{x}(k)
$$

where $\overline{\mathbf{C}}=\mathbf{C}$.

An important assumption that should be satisfied is the observability condition meaning that vibrations of bridge structures are completely observable. The observability condition of a system is considered using the observability matrix (Luenberger 1967), which is definable as

$$
\mathbf{L}=\left[\begin{array}{c}
\overline{\mathbf{C}} \\
\overline{\mathbf{C}} \overline{\mathbf{A}} \\
\vdots \\
\overline{\mathbf{C}}^{p-1}
\end{array}\right]
$$

The observability condition is that the matrix in Eq.(8) should be a rank of $2 n(=m \times p)$. Therein $p$ represents $p$ time spans or $p$ blocks. The discrete state variable in terms of the observability matrix $\mathbf{L}$ is rewritable as $\mathbf{L x}(k)=\hat{\mathbf{x}}(k)$, and substituting into Eqs.(6) and (7). Then the state equation as a block companion form (Luenberger 1967) can be obtained as

$$
\begin{aligned}
& \hat{\mathbf{x}}(k+1)=\hat{\mathbf{A}} \hat{\mathbf{x}}(k)+\hat{\mathbf{B}} \mathbf{f}(k) \\
& \mathbf{y}(k)=\hat{\mathbf{C}} \hat{\mathbf{x}}(k)
\end{aligned}
$$


where the state transition matrix $\hat{\mathbf{A}}$, input influence matrix $\hat{\mathbf{B}}$ and output influence matrix $\hat{\mathbf{C}}$ through an observability transformation are described as

$$
\hat{\mathbf{A}}=\mathbf{L} \overline{\mathbf{A}} \mathbf{L}^{-1}=\left[\begin{array}{ccccc}
\mathbf{0} & \mathbf{I} & \mathbf{0} & \cdots & \mathbf{0} \\
\mathbf{0} & \mathbf{0} & \mathbf{I} & \cdots & \mathbf{0} \\
\vdots & & & \ddots & \vdots \\
\mathbf{0} & \mathbf{0} & \mathbf{0} & & \mathbf{I} \\
-\mathbf{G}_{p} & -\mathbf{G}_{p-1} & -\mathbf{G}_{p-2} & \cdots & -\mathbf{G}_{1}
\end{array}\right]
$$

where $\mathbf{G}_{i} \in \mathbf{R}^{m \times m}$ for $i=1, \cdots, p$.

$$
\begin{aligned}
& \hat{\mathbf{B}}=\mathbf{L} \overline{\mathbf{B}}=\left[\begin{array}{c}
\overline{\mathbf{C}} \overline{\mathbf{B}} \\
\overline{\mathbf{C}} \overline{\mathbf{A}} \overline{\mathbf{B}} \\
\vdots \\
\overline{\mathbf{C}}^{p-1} \overline{\mathbf{A}}^{p}
\end{array}\right]=\left[\begin{array}{c}
\hat{\mathbf{B}}_{1} \\
\hat{\mathbf{B}}_{2} \\
\vdots \\
\hat{\mathbf{B}}_{p}
\end{array}\right] \\
& \hat{\mathbf{C}}=\overline{\mathbf{C}} \mathbf{L}^{-1}=\left[\begin{array}{llll}
\mathbf{I} & \mathbf{0} & \cdots & \mathbf{0}
\end{array}\right], \text { for measuring velocities. }
\end{aligned}
$$

If coefficient matrices from $\mathbf{G}_{1}$ to $\mathbf{G}_{\mathrm{p}}$ of the system matrix $\hat{\mathbf{A}}$ are known, then the eigenvalues and eigenvectors of the matrix $\hat{\mathbf{A}}$ provide the modal information of the system.

\subsection{AR model}

The major purpose of this section is to derive a linear relationship of system outputs combining with the coefficient $\mathbf{G}_{i}$ matrices of Eq.(11). The derivation in the form of MAR model is summarized in this section. The discrete state variable $\hat{\mathbf{x}}(k)$, which has dimension of $m \times p$, can be divided into $p$ time spans $(p$ blocks) as shown in Eq. (14).

$$
\hat{\mathbf{x}}(k)=\left[\begin{array}{lll}
\hat{\mathbf{x}}_{1}(k) & \cdots & \hat{\mathbf{x}}_{p}(k)
\end{array}\right]^{T}
$$

Equation (9), thus, is rewritable as Eq.(15) which is the block companion form.

$$
\left[\begin{array}{c}
\hat{\mathbf{x}}_{1}(k+1) \\
\hat{\mathbf{x}}_{2}(k+1) \\
\vdots \\
\hat{\mathbf{x}}_{p-1}(k+1) \\
\hat{\mathbf{x}}_{p}(k+1)
\end{array}\right]=\left[\begin{array}{ccccc}
\mathbf{0} & \mathbf{I} & \mathbf{0} & \cdots & \mathbf{0} \\
\mathbf{0} & \mathbf{0} & \mathbf{I} & \cdots & \mathbf{0} \\
\vdots & & & \ddots & \vdots \\
\mathbf{0} & \mathbf{0} & \mathbf{0} & & \mathbf{I} \\
-\mathbf{G}_{p} & -\mathbf{G}_{p-1} & -\mathbf{G}_{p-2} & \cdots & -\mathbf{G}_{1}
\end{array}\right]\left[\begin{array}{c}
\hat{\mathbf{x}}_{1}(k) \\
\hat{\mathbf{x}}_{2}(k) \\
\vdots \\
\hat{\mathbf{x}}_{p-1}(k) \\
\hat{\mathbf{x}}_{p}(k)
\end{array}\right]+\left[\begin{array}{c}
\hat{\mathbf{B}}_{1} \\
\hat{\mathbf{B}}_{2} \\
\vdots \\
\hat{\mathbf{B}}_{p-1} \\
\hat{\mathbf{B}}_{p}
\end{array}\right] \mathbf{f}
$$

From Eq.(9) (or Eq.(15)), Eq. (10) and Eq.(13), the following linear relationship can be obtained

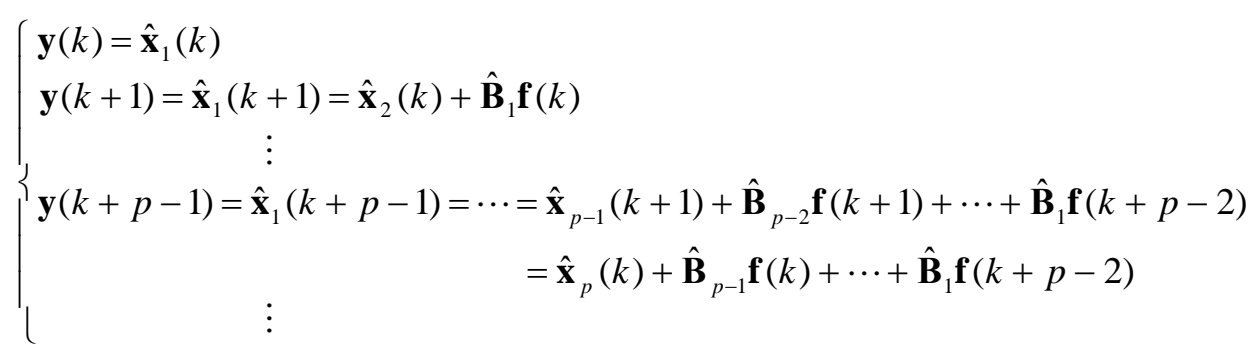

Rearranging the Eq. (16) provides the following linear relationship at each time span. 


$$
\left\{\begin{array}{l}
\hat{\mathbf{x}}_{1}(k)=\mathbf{y}(k) \\
\hat{\mathbf{x}}_{2}(k)=\mathbf{y}(k+1)-\hat{\mathbf{B}}_{1} \mathbf{f}(k) \\
\hat{\mathbf{x}}_{3}(k)=\mathbf{y}(k+2)-\hat{\mathbf{B}}_{1} \mathbf{f}(k+1)-\hat{\mathbf{B}}_{2} \mathbf{f}(k) \\
\vdots \\
\hat{\mathbf{x}}_{p}(k)=\mathbf{y}(k+p-1)-\hat{\mathbf{B}}_{1} \mathbf{f}(k+p-2) \cdots-\hat{\mathbf{B}}_{p-1} \mathbf{f}(k)
\end{array}\right.
$$

Equation (17) is rewritable as

$$
\tilde{\mathbf{x}}(k)=\tilde{\mathbf{y}}(k)-\tilde{\mathbf{B}} \tilde{\mathbf{f}}(k)
$$

where $\widetilde{\mathbf{x}}(k)=\left[\begin{array}{c}\hat{\mathbf{x}}_{p}(k) \\ \vdots \\ \hat{\mathbf{x}}_{1}(k)\end{array}\right] ; \widetilde{\mathbf{y}}(k)=\left[\begin{array}{c}\mathbf{y}(k+p-1) \\ \vdots \\ \mathbf{y}(k)\end{array}\right] ; \widetilde{\mathbf{f}}(k)=\left[\begin{array}{c}\mathbf{f}(k+p-1) \\ \vdots \\ \mathbf{f}(k)\end{array}\right] ;$ and $\widetilde{\mathbf{B}}=\left[\begin{array}{ccccc}\mathbf{0} & \hat{\mathbf{B}}_{1} & \hat{\mathbf{B}}_{2} & \cdots & \hat{\mathbf{B}}_{p-1} \\ \mathbf{0} & \mathbf{0} & \hat{\mathbf{B}}_{1} & \cdots & \hat{\mathbf{B}}_{p-2} \\ \vdots & & & \ddots & \vdots \\ \mathbf{0} & \mathbf{0} & \mathbf{0} & & \hat{\mathbf{B}}_{1} \\ \mathbf{0} & \mathbf{0} & \mathbf{0} & \cdots & \mathbf{0}\end{array}\right]$.

From Eq.(17), the following relationship can be obtained.

$$
\hat{\mathbf{x}}_{p}(k+1)=\mathbf{y}(k+p)-\sum_{i=1}^{p-1} \hat{\mathbf{B}}_{i} \mathbf{f}(k+p-i)
$$

Substituting the linear relation for $\mathbf{x}_{p}(k+1)$ of Eq. (15) into Eq.(19) provides

$$
\mathbf{y}(k+p)=-\mathbf{G} \widetilde{\mathbf{x}}(k)+\hat{\mathbf{B}}_{p} \mathbf{f}(k)+\sum_{i=1}^{p-1} \hat{\mathbf{B}}_{i} \mathbf{f}(k+p-i)
$$

where $\mathbf{G}=\left[\begin{array}{lll}\mathbf{G}_{1} & \cdots & \mathbf{G}_{p}\end{array}\right]$.

The following multivariate ARMA model is obtainable from Eq.(18) and Eq.(20).

$$
\begin{aligned}
& \mathbf{y}(k+p)+\mathbf{G}_{1} \mathbf{y}(k+p-1)+\cdots+\mathbf{G}_{p} \mathbf{y}(k)=\mathbf{D}_{1} \mathbf{f}(k+p-1)+\mathbf{D}_{2} \mathbf{f}(k+p-2)+\cdots \cdots+\mathbf{D}_{p} \mathbf{f}(k) \\
& \mathbf{y}(k)+\mathbf{G}_{1} \mathbf{y}(k-1)+\cdots+\mathbf{G}_{p} \mathbf{y}(k-p)=\mathbf{D}_{1} \mathbf{f}(k-1)+\mathbf{D}_{2} \mathbf{f}(k-2)+\cdots \cdots+\mathbf{D}_{p} \mathbf{f}(k-p) \\
& \mathbf{y}(k)+\sum_{i=1}^{p} \mathbf{G}_{i} \mathbf{y}(k-i)=\sum_{i=1}^{p} \mathbf{D}_{i} \mathbf{f}(k-i)
\end{aligned}
$$

where the coefficient about input $\mathbf{D}_{1} \sim \mathbf{D}_{p}$ can be described as

$$
\left[\begin{array}{c}
\mathbf{D}_{p} \\
\mathbf{D}_{p-1} \\
\mathbf{D}_{p-2} \\
\vdots \\
\mathbf{D}_{1}
\end{array}\right]=\left[\begin{array}{ccccc}
\mathbf{I} & \mathbf{G}_{1} & \cdots & \mathbf{G}_{p-2} & \mathbf{G}_{p-1} \\
\mathbf{0} & \mathbf{I} & \cdots & \mathbf{G}_{p-3} & \mathbf{G}_{p-2} \\
\mathbf{0} & \mathbf{0} & \mathbf{I} & \cdots & \mathbf{G}_{p-3} \\
\vdots & \vdots & . & & \vdots \\
\mathbf{0} & \mathbf{0} & \cdots & \mathbf{0} & \mathbf{I}
\end{array}\right]\left[\begin{array}{c}
\hat{\mathbf{B}}_{p} \\
\hat{\mathbf{B}}_{p-1} \\
\hat{\mathbf{B}}_{p-2} \\
\vdots \\
\hat{\mathbf{B}}_{1}
\end{array}\right]
$$

The AR model with infinite order is equivalent to the ARMA model, which means that one can approximate the responses of a linear system subjected to white-noise input using the AR model with large order (Wang \& Fang 1986; He and De Roeck 1997). The ARMA model in Eq.(21c) can be approximated as AR model as Eq. (23).

$$
\mathbf{y}(k)+\sum_{i=1}^{u} \mathbf{G}_{i} \mathbf{y}(k-i)=\mathbf{e}(k)
$$

where $\mathbf{e}(k)$ denotes a white noise with zero mean and covariance as 


$$
E\left[\mathbf{e}(k+q) \mathbf{e}^{T}(k)\right]= \begin{cases}\boldsymbol{\varepsilon}, & \mathbf{q}=0 \\ \mathbf{0}, & \mathbf{q} \neq 0\end{cases}
$$

Equation (23) is the MAR model derived from the equation of motion of a bridge structure.

\section{AR parameter identification}

Modal parameter identification using the AR model derived in Eq. (23) is possible after estimating AR parameters such as $\mathbf{G}_{p}$. To estimate the AR parameter, firstly we assume the time series considering is a stationary random process, and then a linear equation for the unknown parameter derived from the covariance of the time series is used for estimating the AR parameter.

If $\mathbf{y}(k)$ is a measured signal vector obtained from $m$ measured points, then covariance matrices of $\mathbf{y}(k)$ is obtainable by multiplying each term of Eq. (23) with $\mathbf{y}(k-h)^{T}$ and taking mathematical expectation yields the following AR process for covariance matrix.

$$
\mathbf{R}(h)+\sum_{i=1}^{u} \mathbf{G}_{i} \mathbf{R}(h-i)=\mathrm{E}\left[\mathbf{e}(k) \mathbf{y}(k-h)^{T}\right]= \begin{cases}\mathrm{E}\left[\mathbf{e}(k) \mathbf{y}(k)^{T}\right], & h=0 \\ \mathbf{0}, & h>0\end{cases}
$$

where $\mathrm{E}\left[\mathrm{]}\right.$ indicates the operator for mathematical expectation, and $\mathbf{R}(h)=\mathrm{E}\left[\mathbf{y}(k) \mathbf{y}^{T}(k-h)\right]$ is the covariance matrix of the signal.

It is noteworthy that the white noise $\mathbf{e}(k)$ at the time $k$ is independent with signal $\mathbf{y}(r)$ when $r<k$. Therefore Eq.(25) provides a set of linear equations for $\mathbf{G}_{i}$ during the time $h>0$. To estimate the coefficient matrices $\mathbf{G}_{1}$ to $\mathbf{G}_{u}$ of the system matrix based on the Eq. (25) it needs more than $u$ linear equations. Let $v$ is a number greater than $u$ and start calculation from $h=s+1$ to $h=s+v$, then following linear equations are obtainable.

$$
\mathbf{R g}=\mathbf{r}
$$

where,

$$
\begin{aligned}
& \mathbf{R}=\left[\begin{array}{cccc}
\mathbf{R}(s) & \mathbf{R}(s-1) & \cdots & \mathbf{R}(s-u+1) \\
\mathbf{R}(s+1) & \mathbf{R}(s) & \cdots & \mathbf{R}(s-u+2) \\
\vdots & \vdots & \ddots & \vdots \\
\mathbf{R}(s+u-1) & \mathbf{R}(s+u-2) & \cdots & \mathbf{R}(s) \\
\vdots & \vdots & & \vdots \\
\mathbf{R}(s+v-1) & \mathbf{R}(s+v-2) & \cdots & \mathbf{R}(s+v-u)
\end{array}\right] \\
& \mathbf{g}=\left[\mathbf{G}_{1} ; \mathbf{G}_{2} ; \quad \cdots \quad \mathbf{G}_{u}\right]
\end{aligned}
$$

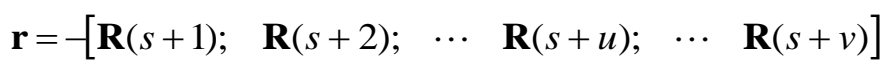

The set of linear equations, called the Yule-Walker equations, can be solved for the AR parameter vector $\mathbf{g}$. The estimated AR parameters from $\mathbf{G}_{1}$ to $\mathbf{G}_{u}$, which are elements of coefficient matrix of $\hat{\mathbf{A}}$, are used for identifying dynamic characteristics of a bridge. The least-squares algorithm is used to solve Eq. (26).

\section{Modal parameter estimation}

The system matrix $\hat{\mathbf{A}}$ is linear transition of the matrix $\overline{\mathbf{A}}$ to satisfy the observability condition. It means that both matrices can be characterized by the same eigensystem. Therefore eigenvalues and vectors from the system matrix $\hat{\mathbf{A}}$ provide modal information about the system A. Modal parameters of a structural system can be obtained solving following eigenvalue problem.

$$
(\overline{\mathbf{A}}-\boldsymbol{\Gamma}) \overline{\mathbf{\Phi}}=\mathbf{0}
$$


where, $\Gamma$ and $\overline{\boldsymbol{\Phi}}$ indicate eigenvalue and eigenvector respectively.

Equation (28) also has following relationships.

$$
\overline{\boldsymbol{\Phi}}^{\mathbf{T}} \overline{\mathbf{A}} \overline{\boldsymbol{\Phi}}=\exp \left(\left[\begin{array}{cc}
\boldsymbol{\lambda} & \mathbf{0} \\
\mathbf{0} & \lambda^{*}
\end{array}\right] \Delta t\right)=e^{\boldsymbol{\Lambda} \Delta t}=\boldsymbol{\Gamma}=\left[\begin{array}{cc}
\boldsymbol{\gamma} & \mathbf{0} \\
\mathbf{0} & \boldsymbol{\gamma}^{*}
\end{array}\right]
$$

where $\gamma_{k}=e^{\lambda_{k} \Delta t}$ and $\gamma_{k}^{*}=e^{\lambda_{k}^{*} \Delta t}$ are the complex conjugate with each other. $\lambda_{k}$ and $\lambda_{k}^{*}$ are the $k$-th order eigenvalues of the state-space equation of $\mathbf{A}$.

$$
\lambda_{k}=-\alpha_{k}+j \beta_{k}=-h_{k} \omega_{k}+j \omega_{k} \sqrt{1-h_{k}^{2}}, \quad \lambda_{k}^{*}=-\alpha_{k}-j \beta_{k}=-h_{k} \omega_{k}-j \omega_{k} \sqrt{1-h_{k}^{2}}
$$

where $\beta_{k}, h_{k}$ and $\omega_{k}$ are the $k$-th order damped natural frequency ( $\left.\mathrm{rad} / \mathrm{s}\right)$, damping constant and undamped natural frequency ( $\mathrm{rad} / \mathrm{s})$, respectively. Therefore using the eigenvalue of $\overline{\mathbf{A}}$, the following relationships are obtainable.

$$
\begin{aligned}
& \beta_{k}=\omega_{k} \sqrt{1-h_{k}^{2}}=\frac{1}{\Delta t} \tan ^{-1}\left|\frac{\gamma_{k}-\gamma_{k}^{*}}{\gamma_{k}+\gamma_{k}^{*}}\right| \\
& \alpha_{k}=h_{k} \omega_{k}=-\frac{1}{2 \Delta t} \ln \left(\gamma_{k} \gamma_{k}^{*}\right)
\end{aligned}
$$

Finally, the $k$-th order undamped natural frequency and damping constant are estimated from Eq.(33) and Eq.(34), respectively.

$$
\begin{aligned}
& \omega_{k}=\sqrt{\alpha_{k}^{2}+\beta_{k}^{2}} \\
& h_{k}=\frac{\alpha_{k}}{\omega_{k}}
\end{aligned}
$$

If $\boldsymbol{\Phi}$ denotes the eigenvector of the observable state transition matrix $\hat{\mathbf{A}}$, then the following relationship exists between Eq.(28) and the eigen equation for $\hat{\mathbf{A}}$ using the observability matrix $\mathbf{L}$.

$$
\left(\mathbf{L} \overline{\mathbf{A}} \mathbf{L}^{-1}-\mathbf{L} \boldsymbol{\Gamma} \mathbf{L}^{-1}\right) \mathbf{L} \overline{\boldsymbol{\Phi}}=(\hat{\mathbf{A}}-\boldsymbol{\Gamma}) \boldsymbol{\Phi}=\mathbf{0}
$$

Therefore, the eigenvector of $\hat{\mathbf{A}}$ can be estimate using the following Eq.(36).

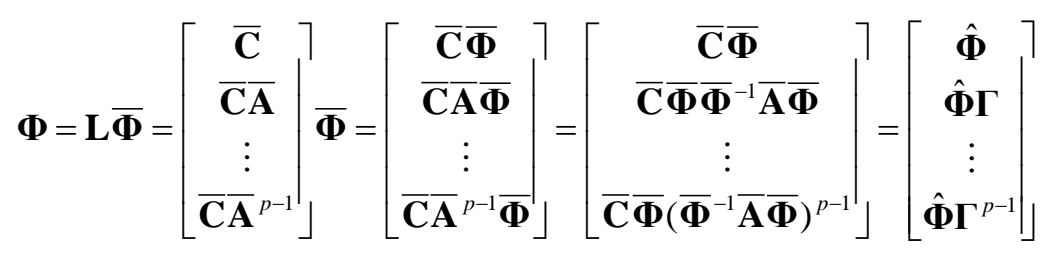

\section{Experiments}

\subsection{Laboratory moving vehicle experiment}

Laboratory experiments for bridge vibrations under a moving vehicle are performed to investigate feasibility of the proposed approach for bridge health monitoring. The experiment setup is shown in Figure 1 with geometry of the girder model that is idealized from a real bridge. Details of the structural property of the girder are also summarized in Figure 1.

Three points at 1/4,1/2, and 3/4 of the span length are the observation points for acceleration responses. The damaged substructure shown in Figure 1 is realized by applying saw cuts at both sides of flanges. The saw cuts result in about $11 \%$ decrease of the bending rigidity of the beam in comparing with that of intact 
beam, which is estimated from measured displacement responses.

[Figure 1]

[Table 1]

As previously mentioned in Introduction, this study focuses on the use of traffic-induced vibrations of bridges even including short span bridges. Moreover, for health monitoring of short span bridges, use of the inspection car which can excite the bridge and process dynamic responses of the bridge while passing on the bridge is another idea of this study. It means that investigating effects of vehicle and vehicle speed are significant matters in health monitoring of short span bridges. Therefore two different vehicle speeds such as $0.93 \mathrm{~m} / \mathrm{s}$ and $1.63 \mathrm{~m} / \mathrm{s}$, which approximate the vehicle speeds of $20 \mathrm{~km} / \mathrm{h}$ and $40 \mathrm{~km} / \mathrm{h}$ on the real bridge with span length of about $40 \mathrm{~m}$ respectively using the speed parameter, are used in the experiment. Two types of vehicle models, which are different dynamic characteristics with each other, are also used in the experiment. The dynamic feature of the vehicle model is changeable using a different set of mass and spring of the vehicle model. Running scenarios of vehicles during the experiment are summarized in Table 1. The fundamental frequencies and damping constants with and without damage are summarized in Table 2. Those fundamental frequencies are obtained from free vibration experiments. On the other hand, damping constants are estimated from the free vibration time histories after the vehicle leaving the model girder because of their dependence on the amplitude. The sampling rate of signals is $100 \mathrm{~Hz}$.

Examples of acceleration time histories of the bridge and their Fourier amplitude spectra before and after damage are shown in Figure 2. It shows that the amplitude of acceleration responses drastically decreases due to the damage. Increasing damping constant due to the saw cuts (see Table 2) may be a major reason of the decreasing of the acceleration amplitude. The appearance of relatively many peaks of dominant frequencies due to the saw cuts in comparison with those of the intact bridge is also observed. Another interesting point is that dominant frequencies near $2.5 \mathrm{~Hz}$ and $23.4 \mathrm{~Hz}$ appearing in the intact bridge are weakened by the damage. That aspect directly links with the identified result by the SAR model, which will be discussed in section 5.2.

[Figure 2]

[Table 2]

[Table 3]

\subsection{Modal parameters of girders}

A two-step identification is conducted to identify modal parameters of the bridge model and to obtain information about a pattern change according to damage. The brief procedure is: 1) the modal parameters relating to dominant frequencies and damping constants are estimated by the SAR model; 2) then the modal parameters including mode vectors are estimated using the MAR model; and 3) select the mode vector of the frequency corresponding to the dominant frequency estimated by the SAR model. 
Modal parameter identification by the SAR model is conducted using experimental data. Those scenarios considering in this study are summarized in Table 3. Dominant frequencies and system damping constants by the SAR model using the data under V1 vehicle running with different speed are summarized in Figure 3 and Figure 4 respectively. Those results under V2 vehicle running are respectively shown in Figures 5 and 6 . In figures identified parameters in the left and right parts respectively indicate those parameters of intact and damaged girders. Mean and CV in figures denote the mean value and coefficient of variance respectively. Therein mean value and CV are basically used for examining statistical properties of the identified result. This study also adopts mean value and CV as parameters indicating change of bridges' health condition. Data numbers of the second frequency missing correspond to observation data at the span center which seldom provides the second bending mode.

[Figure 3]

[Figure 4]

[Figure 5]

[Figure 6]

Observations demonstrate that the lower speed scenarios give very similar results with those from free vibration experiment. The mean values of identified frequencies of the intact girder of SCN1 are $2.61 \mathrm{~Hz}$, $9.76 \mathrm{~Hz}$ and $23.4 \mathrm{~Hz}$ (see the left part of Figure 3), which link with $2.6 \mathrm{~Hz}, 10.6 \mathrm{~Hz}$ and $23.3 \mathrm{~Hz}$ for the first, second and third modes obtained from the free vibration respectively. Those frequencies after introducing the damage are $2.53 \mathrm{~Hz}, 11.12 \mathrm{~Hz}$ and $23.07 \mathrm{~Hz}$ as shown in the right part of Figure 3 , which are corresponding to $2.5 \mathrm{~Hz}, 9.7 \mathrm{~Hz}$ and $23.1 \mathrm{~Hz}$ from the free vibration of the damage girder respectively. On the other hand, those identified results under vehicle of higher speed (SCN2) are greatly biased from parameters obtained from free vibrations as shown in Figure 4. Similar tendency is observed under V2 vehicle running scenarios such as SCN3 and SCN4 respectively shown in Figures 5 and 6. The results demonstrate that the effect of the vehicle system to bridge vibrations, so called traffic-induced vibration of bridges or non-stationary vibration, increases with increasing speed, and as a result the identified results under higher vehicle speed yield more biased identification results than those of lower speed. For the first frequencies in any scenarios, however, mean values tend to decrease on the other hands CVs tend to increase due the damage.

An interesting point is that the second frequency of the damaged girder is greater than that of the intact girder. This is different from the well known fact that decreasing stiffness results in lower frequencies. One of the reasons for the result is that the dominant frequency of the intact girder is disturbed by damages, and, as a result, the dominant frequencies both near $11 \mathrm{~Hz}$ as well as $9.5 \mathrm{~Hz}$ of the damaged girder are detected, which can be verified comparing the PSD curve of raw data and with that curve reconstructed by identified AR coefficient of the SAR model as shown in Figure 7. Therein, the B' and C' are identified dominant frequencies of the damaged girder, in which $\mathrm{B}$ and $\mathrm{C}$ are those frequencies of the intact girder. Similarly the damage gives additional dominant frequency near $20 \mathrm{~Hz}$ except $23.2 \mathrm{~Hz}$ that is about the third dominant frequency of the intact girder. 
Usually the damping constants derived from eigenvalue of system matrix $\mathbf{A}$ may be subject to appreciable error (Pappa \& Ibrahim 1981), and as a result larger coefficient of variance than that of the identified frequency is observed. However, despite of their appreciable error the pattern change of identified damping constants due to the damage is very apparent comparing to that of the dominant frequency as shown in Figures 3, 4, 5 and 6.

Observations from the identified results show that the damage causes disturbance, like mean value and $\mathrm{CV}$, of identified dominant frequency and system damping constant regardless of vehicle speed and type. This is the reason why this study is even focusing on the pattern change of modal parameters to acquire additional information about current health condition of the bridge.

For further information, identified results under both V1 and V2 vehicles running lower speed are summarized in Figure 8, which also demonstrates pattern change of identified modal parameters. Figure 9 shows the plot of all the identified results. It also shows clear pattern change of identified modal parameters despite of their severe variation of identified parameters. It demonstrates that the identified system modal parameters using the traffic-induced vibration data under a given moving vehicle like an inspection car, which are usually affected by external dynamic sources such as vehicle's dynamic system and as a result are not directly connected with natural modal parameters of the bridge itself, can provide information for bridge's health condition.

[Figure 8]

[Figure 9]

The identified mode shapes of the intact girder from the data under the vehicle moving with the low speed (SCN5) using the MAR model are shown in Figure 10a), in which the average mode vector at each observation point is compared with that obtained by the FE analysis. The correlation of mode shapes obtained from the MAR model and FE analysis is evaluated using the index of modal assurance criterion (MAC) (Allemang \& Brown 1983). If two corresponding modes are well correlated the MAC value is close to one. The MAC is defined as

$$
M A C_{i, i}=\frac{\left|\boldsymbol{\Phi}_{i I}^{T} \boldsymbol{\Phi}_{i A}\right|^{2}}{\boldsymbol{\Phi}_{i I}^{T} \boldsymbol{\Phi}_{i I} \boldsymbol{\Phi}_{i A}^{T} \boldsymbol{\Phi}_{i A}}
$$

where $\boldsymbol{\Phi}_{i I}$ and $\boldsymbol{\Phi}_{i A}$ represents two mode vectors of the $i$-th mode estimated from different method.

The MAC values of three modes shown in Figure 10a) are very close to one. It demonstrates that the identified mode shapes of the intact girder are well correlated with analytical ones. The mode shapes before and after the damage are shown in Figure 10b). MAC values of 0.98 and 0.97 respectively for the first and second modes indicate changes of bridge's health condition.

[Figure 10]

[Table 4]

[Table 5] 
For information the modal parameters identified using the MAR model under vehicles running with lower speed $(0.93 \mathrm{~m} / \mathrm{s})$ are summarized in Table 4 and Table 5 with those taken from the SAR model. For identified frequencies, there is no great difference between SAR and MAR model. On the other hand relatively great difference is observed for identified system damping constants of the damaged girder between two models.

Observations from the experimental investigation demonstrate the feasibility of the AR model for modal identification of short span bridges under a vehicle with a low speed. Moreover comparing the identification results between intact and damage girders such as pattern changes of identified parameters encourages the use of the method for long term health monitoring even for short span bridges.

\section{Conclusions}

In this study, feasibility of modal parameter identification for health monitoring of short span bridges from traffic-induced vibrations is investigated through a laboratory running vehicle experiment. A two-step identification is conducted in this study to identify modal parameters of the bridge model and to obtain information about a pattern change according to damage more clearly: the first step identifies modal frequencies and damping using a SAR model; and identifying mode shapes by means of MAR model is the second step.

Observations demonstrate that the dominant frequency and damping constant of the bridge are well identified using the measurement data under vehicle running with lower speed. Reasonable identification results for mode vectors are also observed. Moreover comparing the identification results between intact and damage girders such as pattern change of identified parameters encourages the use of the method for long term health monitoring even for short span bridges. Among three modal parameters such as frequency, damping constant and mode vector, the damping constant is the most sensitive parameter to the damage. Therefore, the pattern change of the identified damping constant due to damage may act as a good indicator of bridge's health condition despite of its appreciable error in identification. Moreover the change of mode vectors due to the damage is also observed despite of using limit numbers of sensors.

The procedure or algorithm discussed in this study can be embedded in wireless sensor nodes, and those modal parameters automatically detected at each sensor node are applicable for monitoring bridges' health condition. How to assess health condition of the bridges using the identified modal parameters quantitatively is a next step for this study.

\section{Acknowledgements}

The supports of the Japan Society for the Promotion of Science (Grant-in-Aid for Exploratory Research under project No.19656112 and Grant-in-Aid for Scientific Research (C) under project No. 20560443) are gratefully acknowledged.

\section{References}

Allemang, R.L. \& Brown, D.L. 1983. A correlation coefficient for modal vector analysis. Proc. of the First Int. Modal Analysis Conference, Bethel, Connecticut, USA, 690-695.

Doebling, S.W., Farrar, C.R., Prime, M.B. \& Shevitz, D.W. 1996. Damage identification and health monitoring of structural and mechanical systems from changes in their vibration characteristics: A literature review. Los Alamos National Laboratory Report LA-3070-MS.

Gersch, W., Nielsen, N.N. \& Akaike, H. 1973. Maximum likelihood estimation of structural parameters from random vibration data. J. of Sound and Vibration, 31(3), 295-308.

Friswell, M.I. \& Mottershead, J.E. 1994. Finite element model updating in structural dynamics. Kluwer Academic Publishers, 56-77.

He, X. \& De Roeck, G. 1997. System identification of mechanical structures by a high-order multivariate autoregressive model. Computers and Structures, 64(1-4), 341-351. 
Hoshiya, M. \& Saito, E. 1984. Structural identification by extended Kalman filter, J. Engg. Mech., ASCE, 110(12), 1757-1770.

Kim, C.W., Kawatani, M., \& Kim, K.B. 2005. Three-dimensional dynamic analysis for bridge-vehicle interaction with roadway roughness, Computers and Structures, 83(19-20), 1627-1645.

Kim, C.W. \& Kawatani, M. 2008. Pseudo-static approach for damage identification of bridges based on coupling vibration with a moving vehicle. Structure and Infrastructure Engineering, 4(5), 371-379.

Luenberger, D.G. 1967. Canonical from for linear multivariate systems. IEEE, Transactions on. Automatic Control, AC-12, 290-293.

MATLAB 1997. The Math Works, Inc. Natick, Massachusetts.

Pappa, R.S. \& Ibrahim, S.R. 1981. A parametric study of the Ibrahim time domain modal identification algorithm. Shock and Vibration Bulletin, No.51, Part 3, pp.43-72.

Shinozuka, M., Yun C.B. \& Imai, H. 1982. Identification of linear structural dynamic systems. J. Engg. Mech. Div., ASCE, 108(6), 1371-1390.

Wang, Z. \& Fang, T. 1986. A time-domain method for identifying modal parameters. J. Appl. Mech., ASME, 53(3), 28-32. 

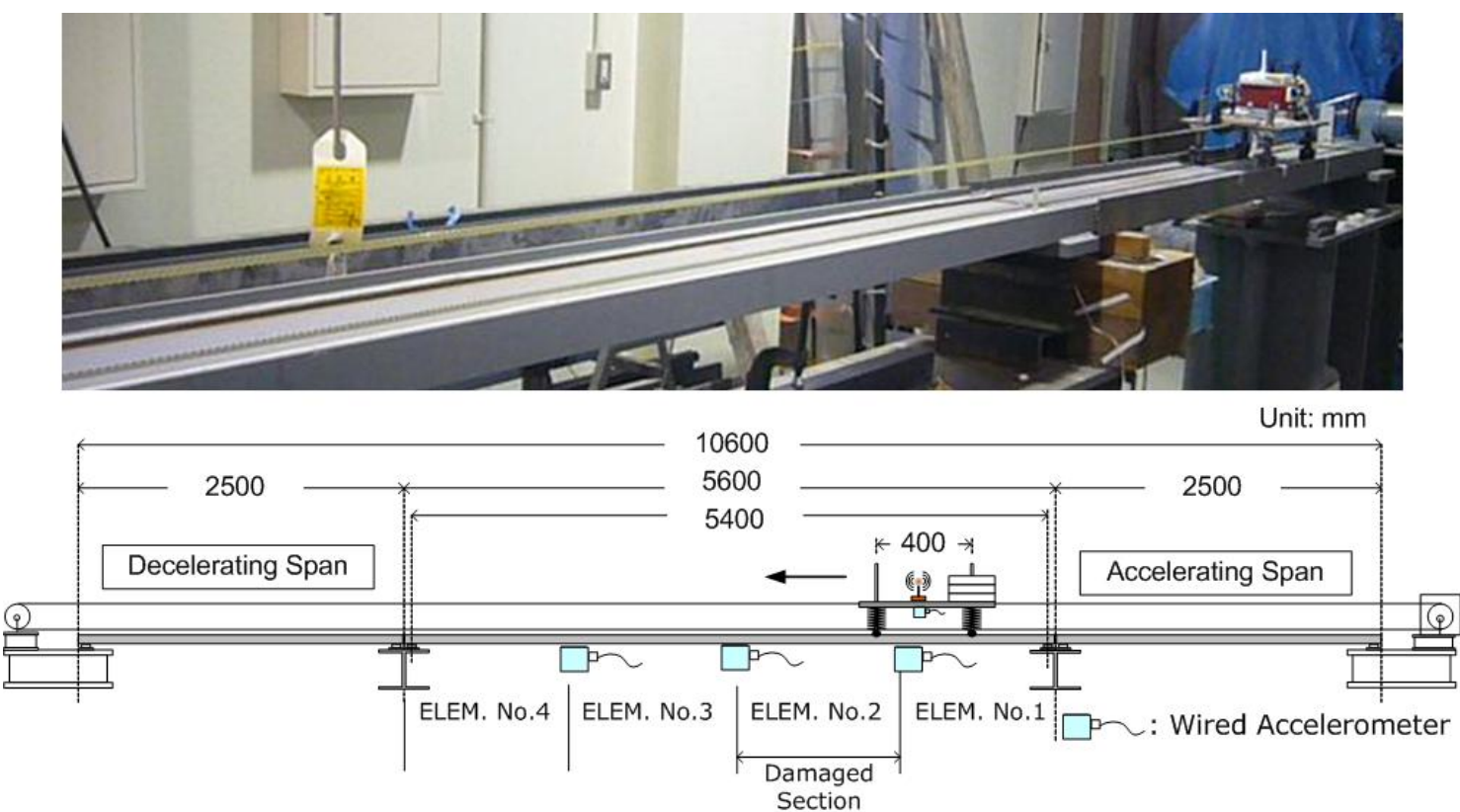

a)

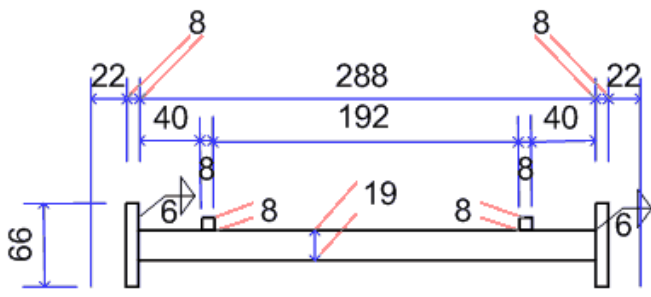

Material properties

$E=2.1 \mathrm{E} 06\left(\mathrm{kgf} / \mathrm{cm}^{2}\right)$ $w=7.8 \mathrm{E}-3\left(\mathrm{kgf} / \mathrm{cm}^{3}\right)$

b)

Cross-section

Sectional properties $A=66.56 \mathrm{~cm}^{2}$ $I=55.67 \mathrm{~m}^{4}$

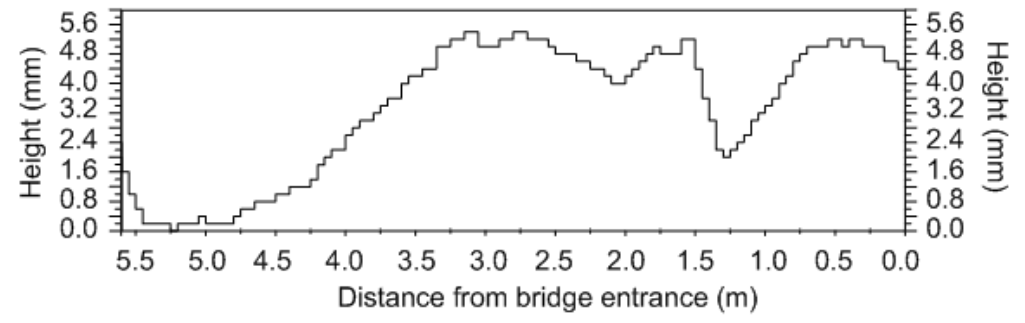

c)

Roadway surface profile

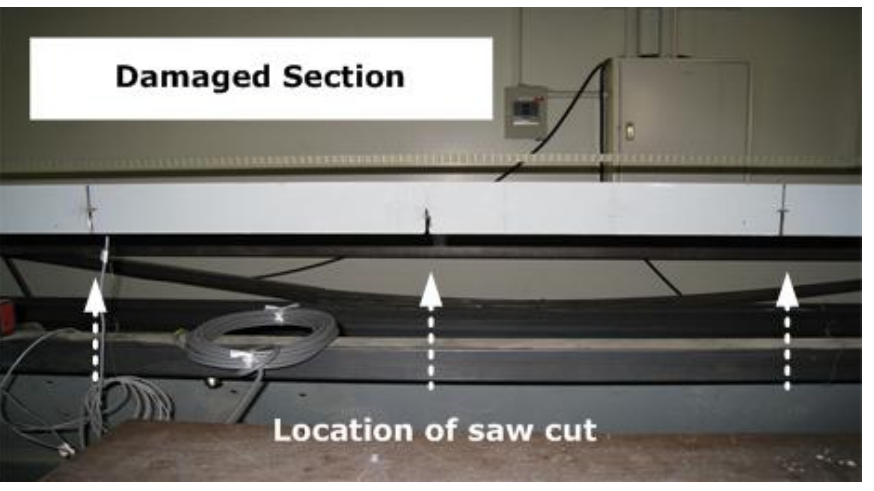

Figure 1. Experimental setup: a) layout of experiment; b) cross section of experiment girder; c) roadway surface profile; d) damaged section. 

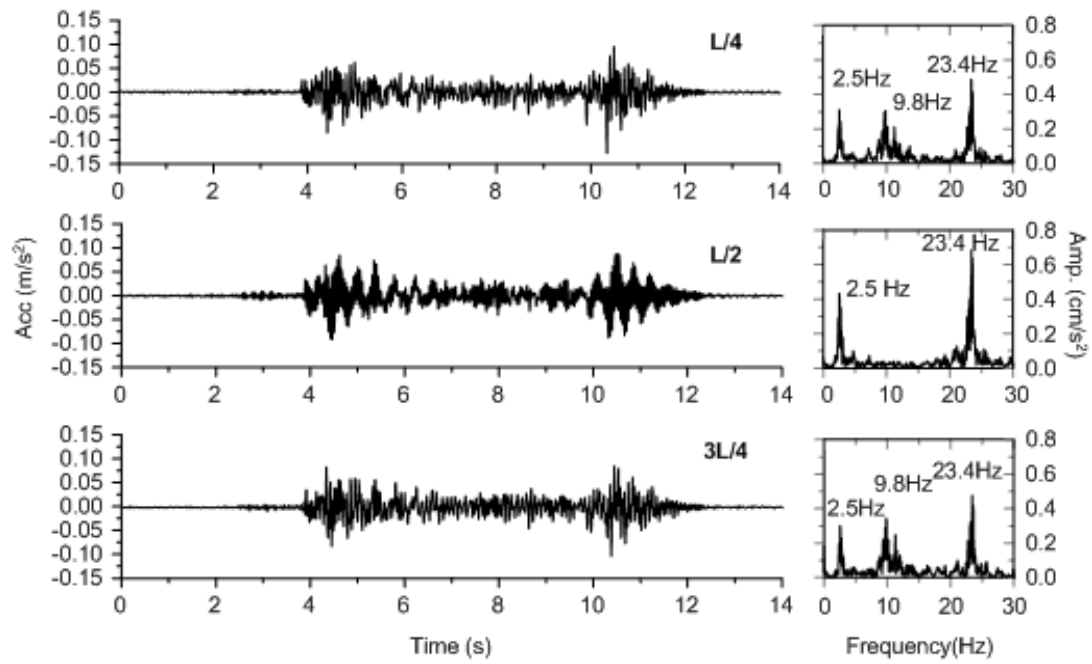

a)
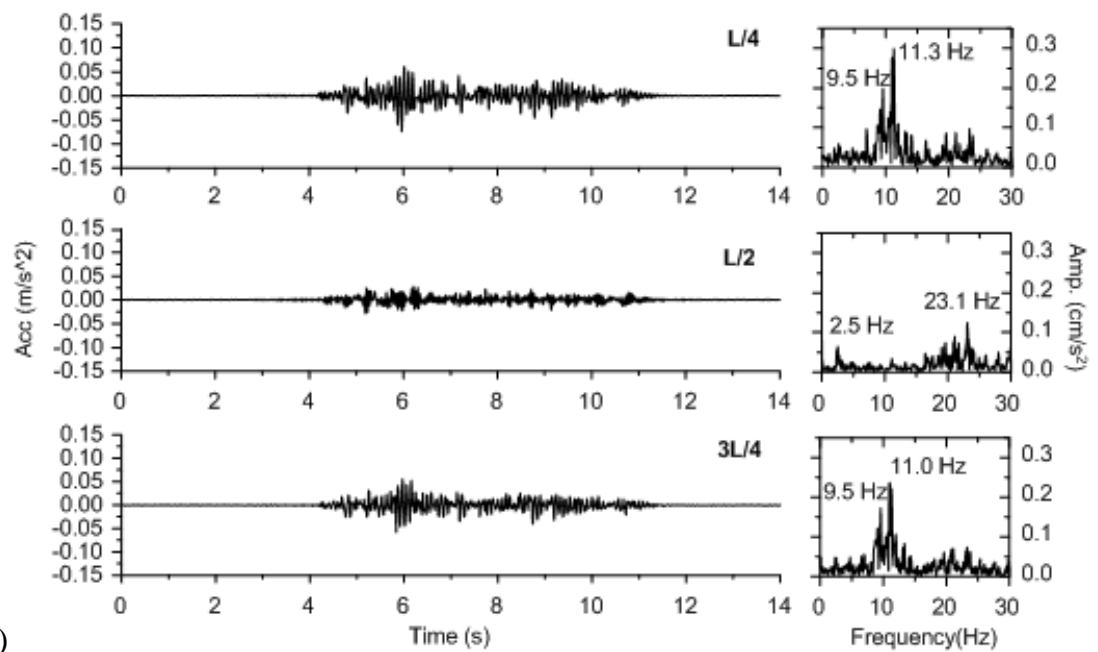

b)

Figure 2. Accelerations of the bridge under V1 vehicle with speed of $0.93 \mathrm{~m} / \mathrm{s}$ : a) Intact; and b) Damaged. 


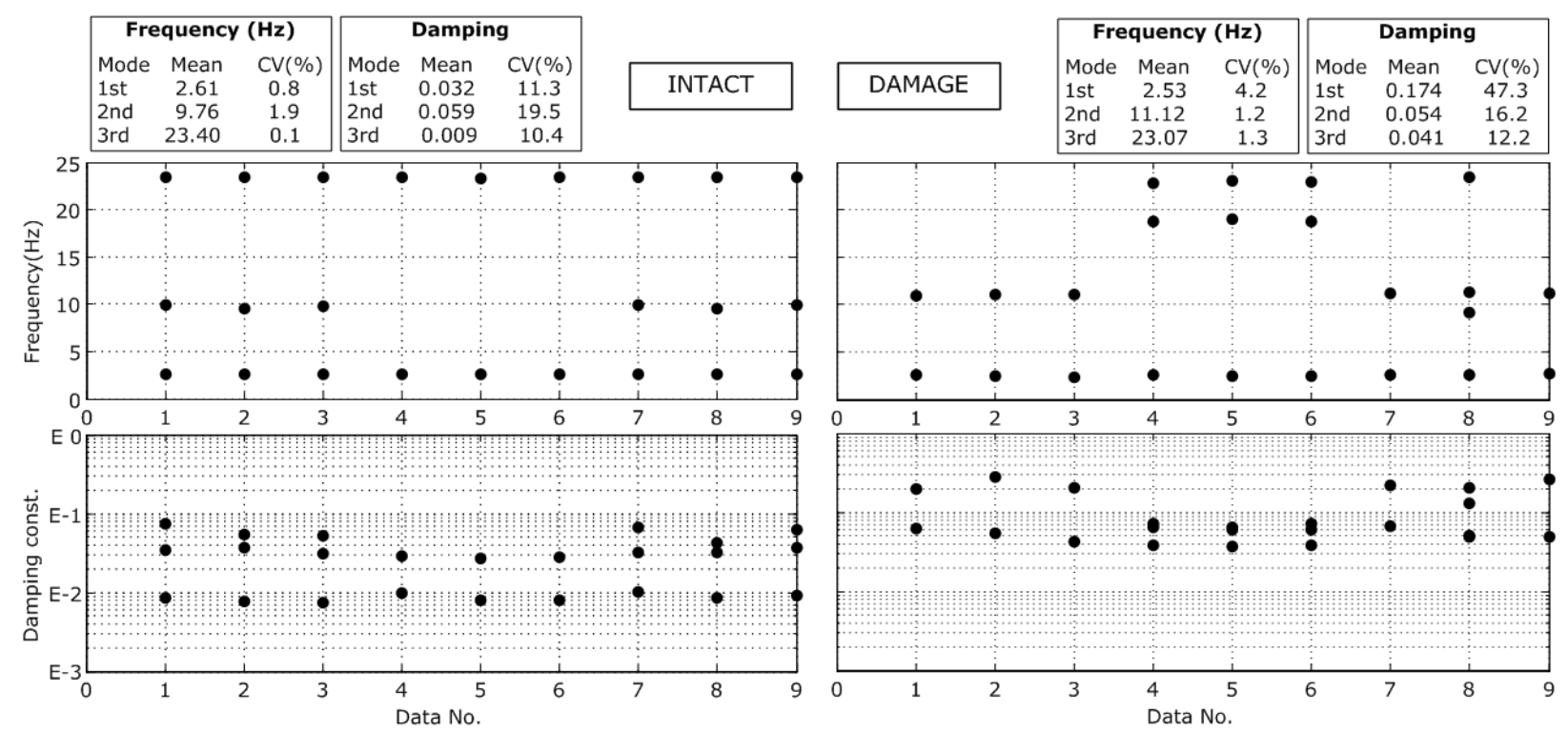

Figure 3. Identified frequencies and damping constants of intact and damaged girders under SCN1; V1 vehicle running with speed of $0.93 \mathrm{~m} / \mathrm{s}$. 


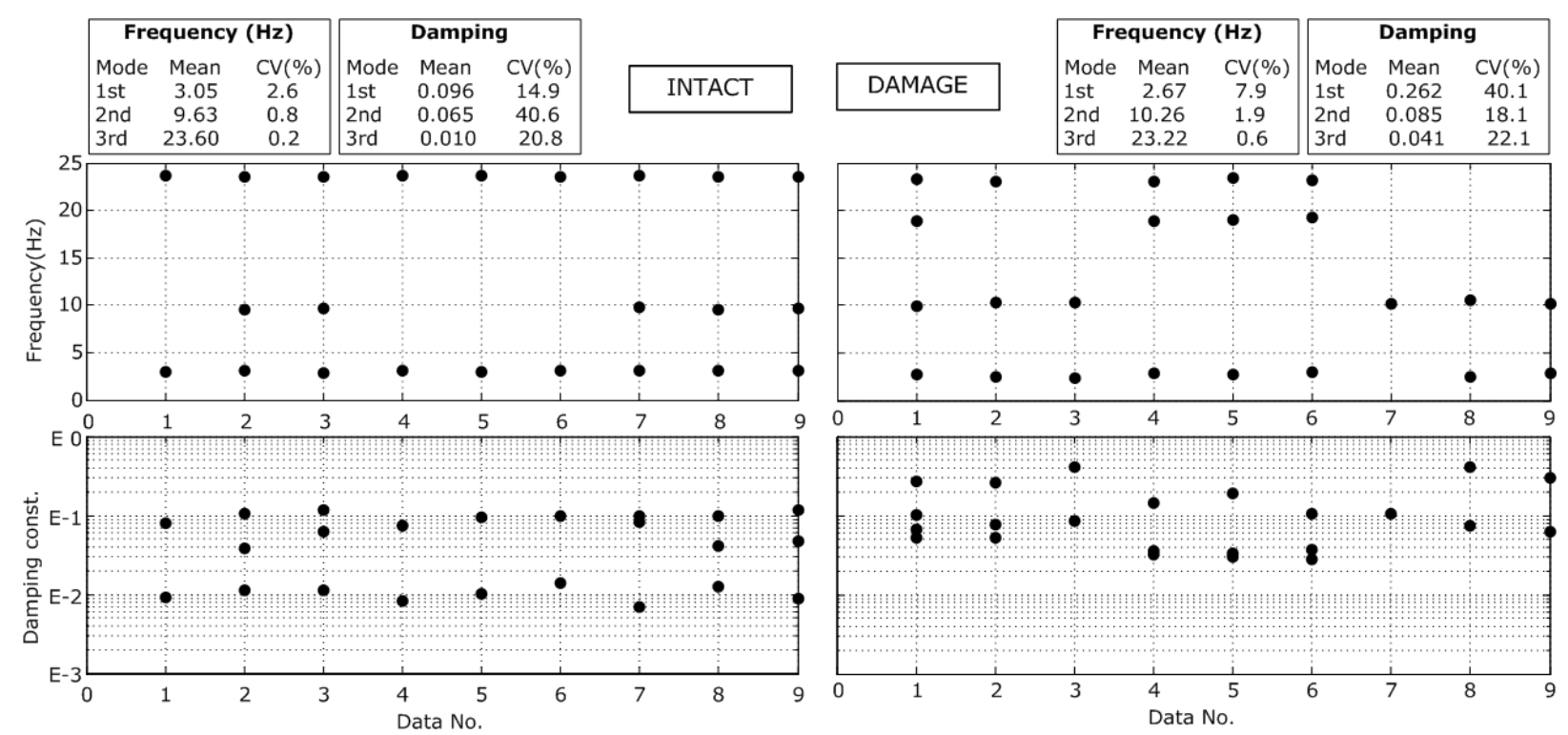

Figure 4. Identified frequencies and damping constants of intact and damaged girders under SCN2; V1 vehicle running with speed of $1.63 \mathrm{~m} / \mathrm{s}$. 

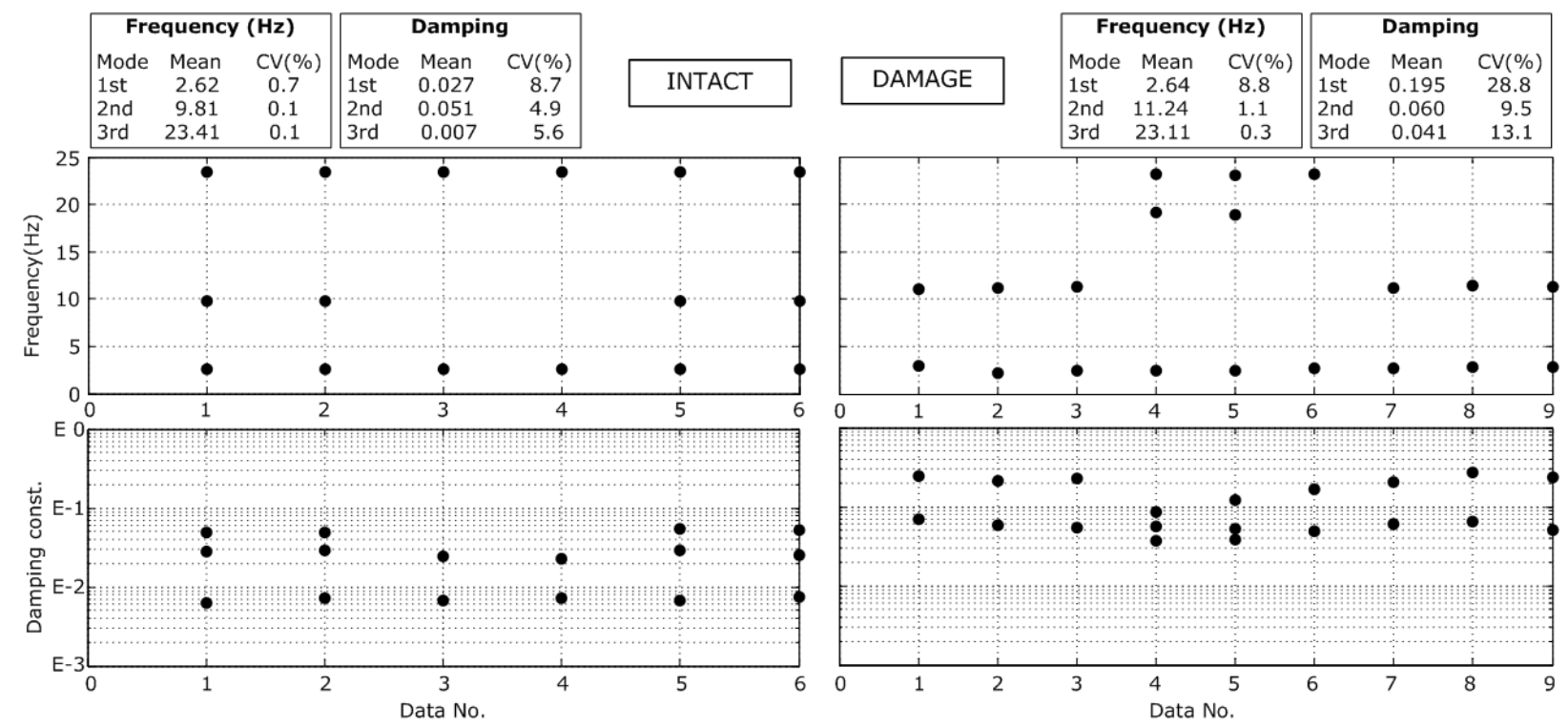

Figure 5. Identified frequencies and damping constants of intact and damaged girders under SCN3; V2 vehicle running with speed of $0.93 \mathrm{~m} / \mathrm{s}$. 

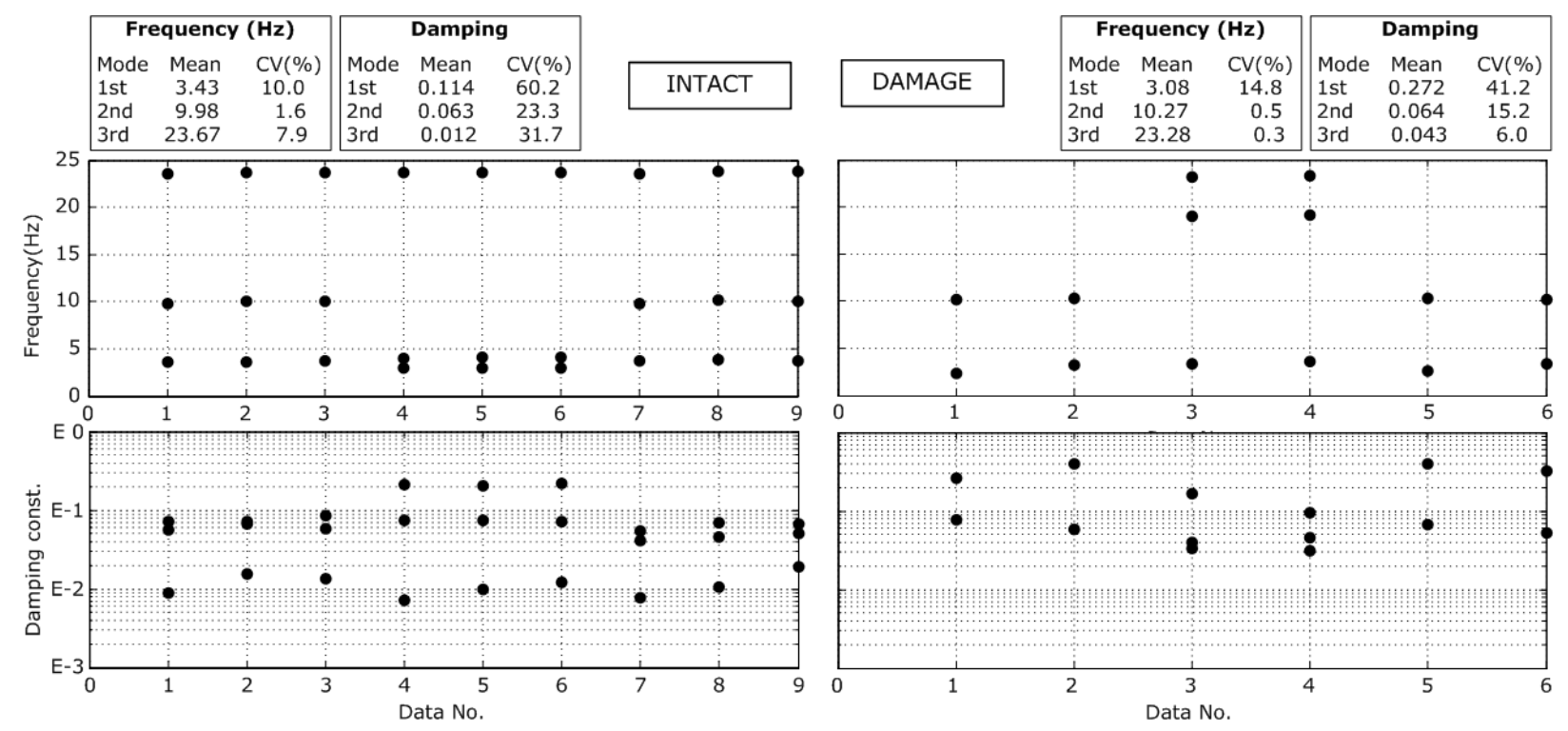

Figure 6. Identified frequencies and damping constants of intact and damaged girders under SCN4; V2 vehicle running with speed of $1.63 \mathrm{~m} / \mathrm{s}$. 
a)
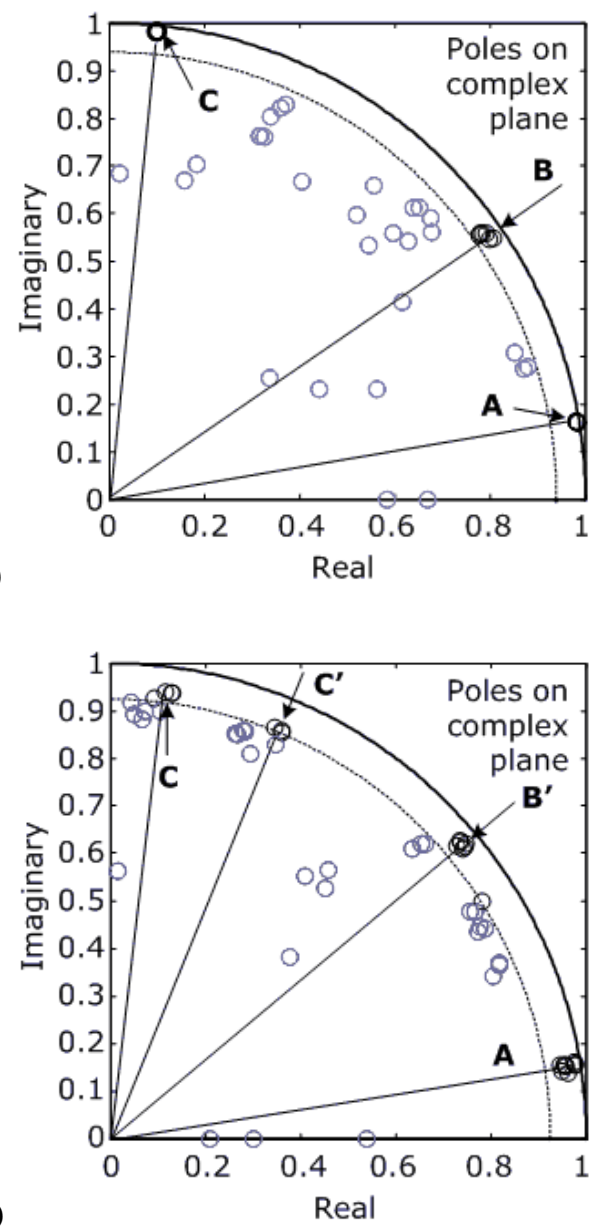
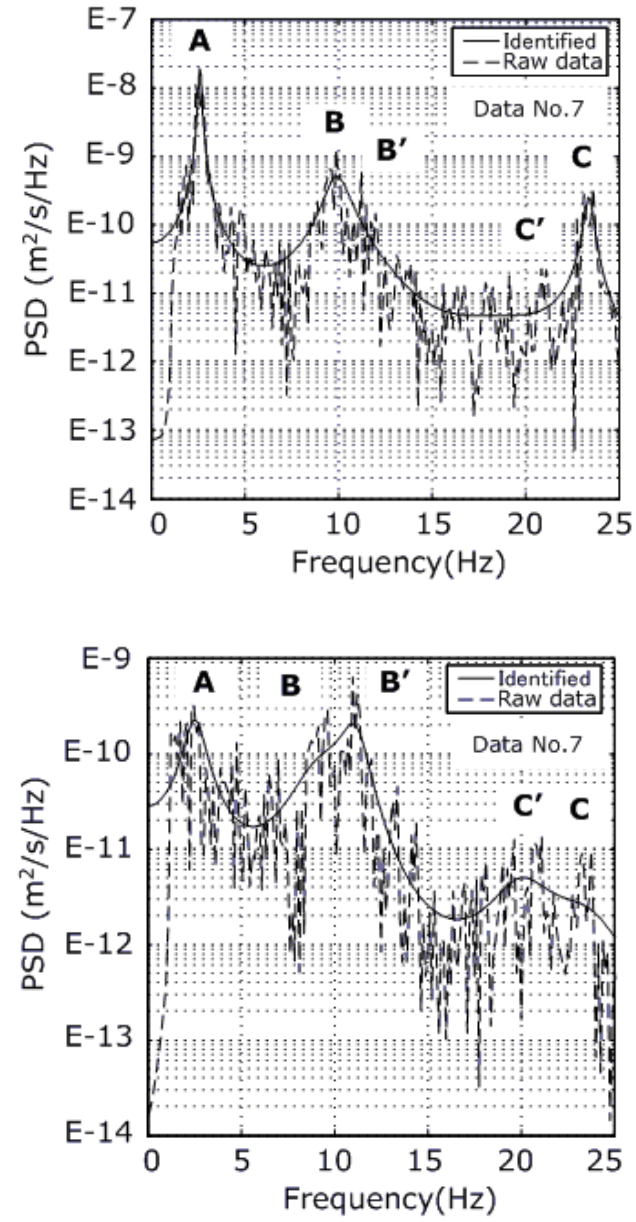

Figure 7 Identified pole plots of SCN1 on complex plane and PSD of Data No. 7; a) Intact; and b) damage 


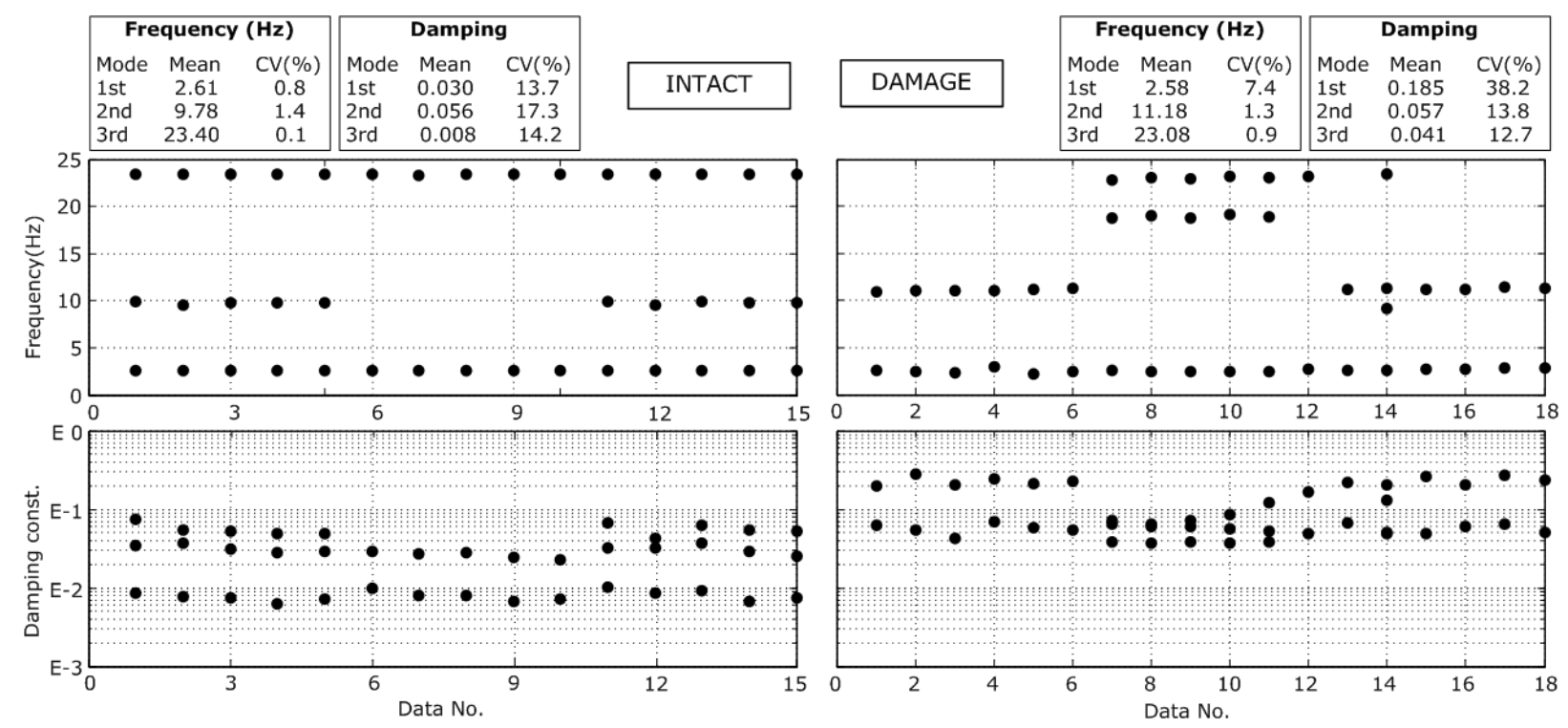

Figure 8. Identified frequencies and damping constants of intact and damaged girders under SCN5; both V1 and V2 vehicles running with speed of $0.93 \mathrm{~m} / \mathrm{s}$. 


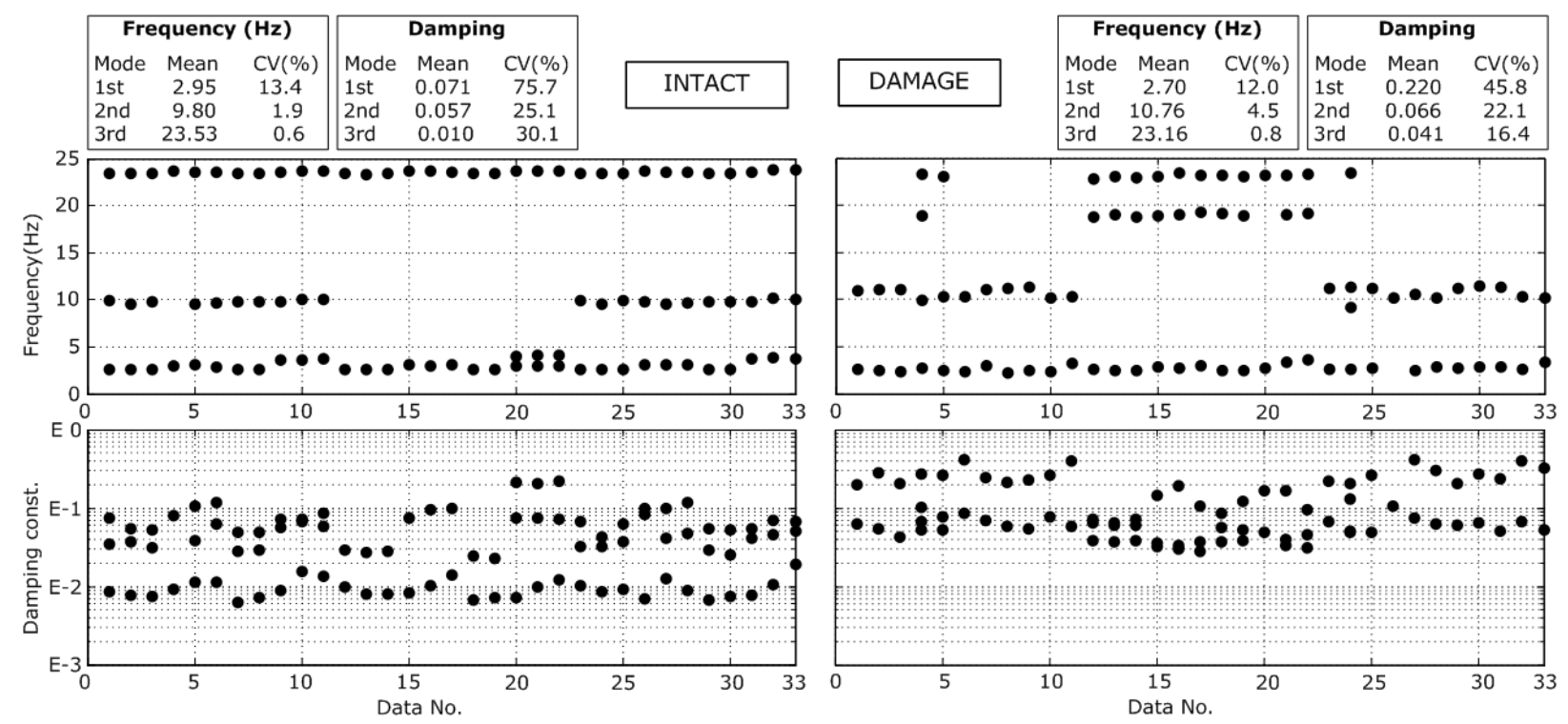

Figure 9. Identified frequencies and damping constants of intact and damaged girders under SCN6; V1 and V2 vehicles running with speed of $0.93 \mathrm{~m} / \mathrm{s}$ and $1.63 \mathrm{~m} / \mathrm{s}$. 


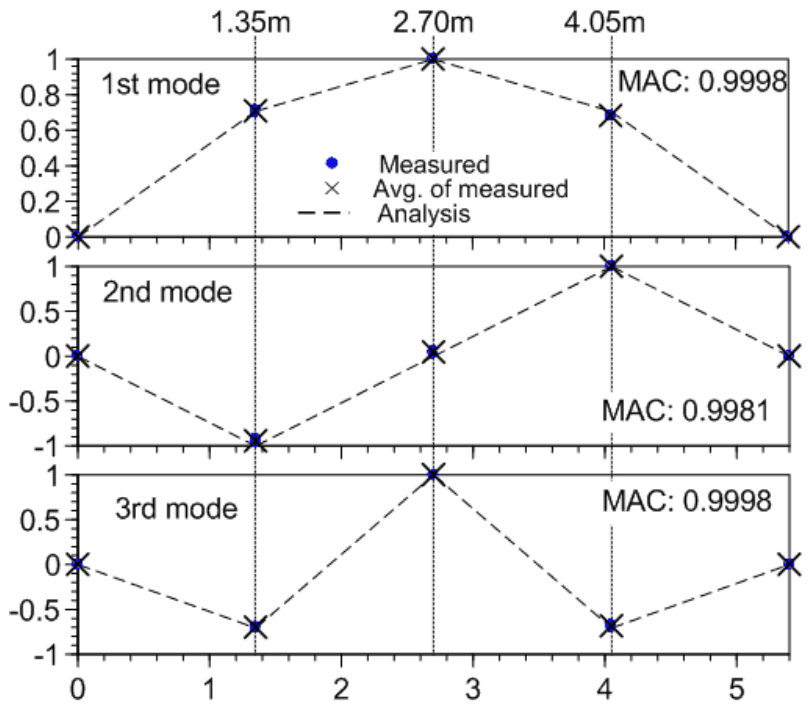

a)

Distance $(\mathrm{m})$
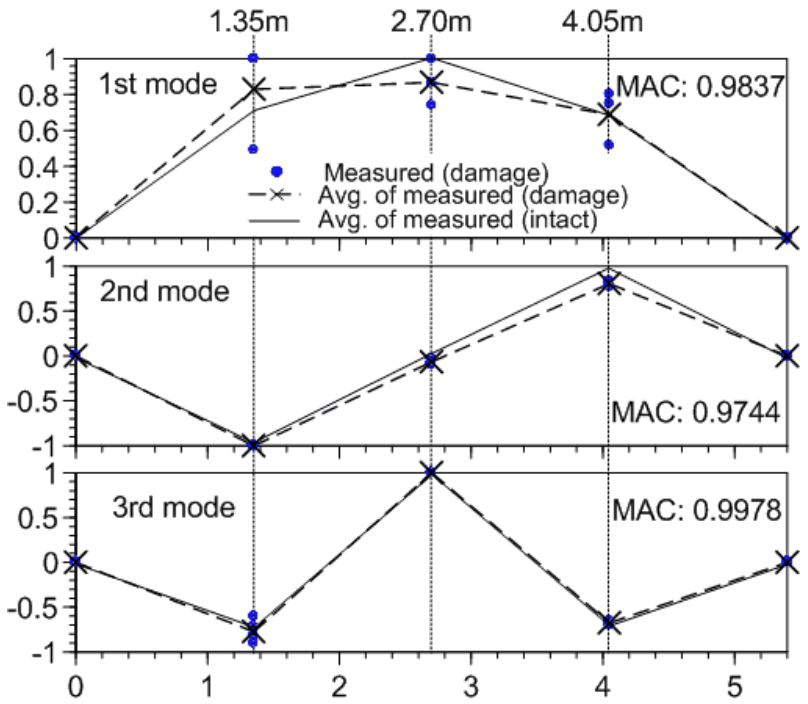

b)

Distance $(\mathrm{m})$

Figure 10. Mode shapes of intact bridge: a) Averaged modes from measured data of intact girder with analytical results; and b) Averaged modes from the measured data of intact and damaged girders respectively. 
Table 1. Vehicle running scenarios during experiment.

\begin{tabular}{cccc}
\hline Vehicle type & Speed $(\mathrm{m} / \mathrm{s})$ & Number of experiment (Intact) & Number of experiment (damage) \\
\hline \multirow{2}{*}{ V1 } & 0.93 & 3 & 3 \\
& 1.63 & 3 & 3 \\
V2 & 0.93 & 2 & 3 \\
& 1.63 & 3 & 2 \\
\hline
\end{tabular}

V1: vehicle with fundamental frequencies for bounce motion of $2.93 \mathrm{~Hz}$ at front and rear axles

V2: vehicle with fundamental frequencies for bounce motion of $3.71 \mathrm{~Hz}$ and $3.81 \mathrm{~Hz}$ at front and rear axles respectively. 
Table 2. Fundamental frequencies and damping constants of the bridge model.

\begin{tabular}{ccccc}
\hline \multirow{2}{*}{ Mode } & \multicolumn{2}{c}{ Intact } & \multicolumn{2}{c}{ Damage } \\
& Frequency $(\mathrm{Hz})$ & Damping constant & Frequency $(\mathrm{Hz})$ & Damping constant \\
\hline First & 2.6 & 0.058 & 2.5 & 0.185 \\
Second & 10.6 & - & 9.7 & - \\
Third & 23.3 & - & 23.1 & - \\
\hline
\end{tabular}


Table 3. Considered scenarios

\begin{tabular}{l|l}
\hline Scenario & Remarks \\
\hline SCN1 & V1 vehicle running with speed of $0.93 \mathrm{~m} / \mathrm{s}$ \\
SCN2 & V1 vehicle running with speed of $1.63 \mathrm{~m} / \mathrm{s}$ \\
$\mathrm{SCN} 3$ & V2 vehicle running with speed of $0.93 \mathrm{~m} / \mathrm{s}$ \\
$\mathrm{SCN} 4$ & V2 vehicle running with speed of $1.63 \mathrm{~m} / \mathrm{s}$ \\
$\mathrm{SCN} 5$ & Considering the all vehicle running scenario with speed of $0.93 \mathrm{~m} / \mathrm{s}(\mathrm{SCN} 1$ and SCN3) \\
SCN6 & Considering all the running scenarios (from SCN1 to SCN4) \\
\hline
\end{tabular}


Table 4. Identified mean dominant frequencies by SAR and MAR model using the data of SCN5

\begin{tabular}{lllll}
\hline & Intact & & Damaged & MAR \\
\hline First & SAR & MAR & SAR & 2.57 \\
Second & 2.61 & 2.56 & 2.58 & 11.27 \\
Third & 9.78 & 9.70 & 11.18 & 23.26 \\
\hline
\end{tabular}


Table 5. Identified mean system damping constants by SAR and MAR model using the data of SCN5

\begin{tabular}{lllll}
\hline & Intact & & Damaged & MAR \\
\hline SArst & SAR & MAR & SAR & 0.073 \\
Second & 0.030 & 0.020 & 0.185 & 0.036 \\
Third & 0.056 & 0.040 & 0.057 & 0.012 \\
\hline
\end{tabular}

